The $Q_{i}$ site of cytochrome $b$ is a promiscuous drug target in Trypanosoma cruzi and

\title{
Leishmania donovani
}

Richard J. Wall1,2, Sandra Carvalho ${ }^{1,2}$, Rachel Milne' ${ }^{2}$, Juan A. Bueren-Calabuig ${ }^{3}$, Sonia Moniz², Juan Cantizani-Perez ${ }^{4}$, Lorna MacLean ${ }^{3}$, Albane Kessler ${ }^{4}$, Ignacio Cotillo ${ }^{4}$ Lalitha Sastry ${ }^{3}$, Sujatha Manthri3 ${ }^{3}$, Stephen Patterson ${ }^{2}$, Fabio Zuccotto ${ }^{3}$, Stephen Thompson ${ }^{3}$, Julio Martin ${ }^{4}$, Maria Marco ${ }^{4}$, Timothy J. Miles ${ }^{4}$ Manu De Rycker ${ }^{3}$, Michael G. Thomas ${ }^{3}$, Alan H. Fairlamb², lan H. Gilbert ${ }^{3}$ and Susan Wyllie ${ }^{2 *}$

${ }^{1}$ Authors contributed equally

2 Division of Biological Chemistry and Drug Discovery, Wellcome Centre for Anti-Infectives Research, School of Life Sciences, University of Dundee, Dow Street, Dundee DD1 5EH, United Kingdom.

${ }^{3}$ Drug Discovery Unit, Wellcome Centre for Anti-Infectives Research, School of Life Sciences, University of Dundee, Dow Street, Dundee DD1 5EH, United Kingdom.

${ }^{4}$ Global Health R\&D, GlaxoSmithKline, Tres Cantos, 28760, Spain

Running title: Cyt $b$ - a promiscuous drug target in kinetoplastids

*Corresponding author: Dr Susan Wyllie, Division of Biological Chemistry and Drug Discovery, Wellcome Centre for Anti-Infectives Research, School of Life Sciences, University of Dundee, Dow Street, Dundee DD1 5EH, United Kingdom.

Tel: (44)1382 38 5761; Email: s.wyllie@dundee.ac.uk 


\section{Supplementary figures}

Figure S1 - resistance generation in vitro.

Figure S2 - CNV analysis for $L$. donovani $(L d)$ and $T$. cruzi $(T C)$ clones resistant to compounds 1 and compound 2.

Figure S3 - representative complex III and $\mathrm{O}_{2}$ consumption assay data.

Figure S4 - x-ray structures of cytochrome $b$ in complex with ligands binding to the $Q_{i}$ site.

Figure $\mathrm{S} 5$ - binding mode of antimycin $\mathrm{A}$ in the cytochrome $b \mathrm{Q}_{\mathrm{i}}$ site.

Figure S6 - structural analysis of compound 1 - cytochrome $b$ interactions.

Figure S7 - structural analysis of compound 2 - cytochrome $b$ interactions.

Figure S8 - structural analysis of compound 3 - cytochrome $b$ interactions.

Figure S9 - cytochrome $b$ resistant panel screen.

Figure S10 - complex III assays with compounds identified via the cytochrome $b$ resistant panel screen.

\section{Supplementary tables}

Table S1 - whole genome sequencing for $L$. donovani clones resistant to compound 1 .

Table S2 - whole genome sequencing for T. cruzi clones resistant to compound 1.

Table S3 - compounds 1 and $\mathbf{2}$ in complex III assays with WT and resistant cell lysates.

Table S4 - whole genome sequencing for $L$. donovani clones resistant to compound 2.

Table S5 - compound potencies against wild-type and resistant $L$. donovani cell lines in intra-macrophage assays. 

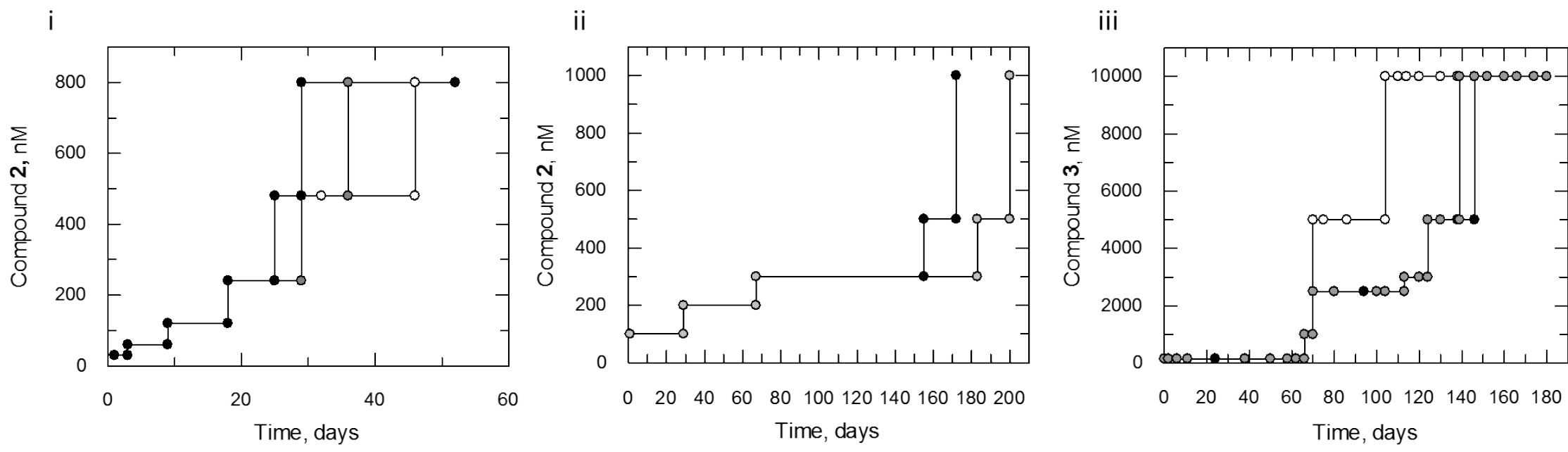

Figure S1 - Resistance generation in vitro. Schematic representation of the generation of compound 2-resistant cell lines in $L$. donovani (promastigotes) (i) and T. cruzi (epimastigotes) (ii) and with compound $\mathbf{3}$ in $T$. cruzi (iii). Each passage of cells in culture (circles) is indicated with clones I, II and III indicated in black, white and grey, respectively. 


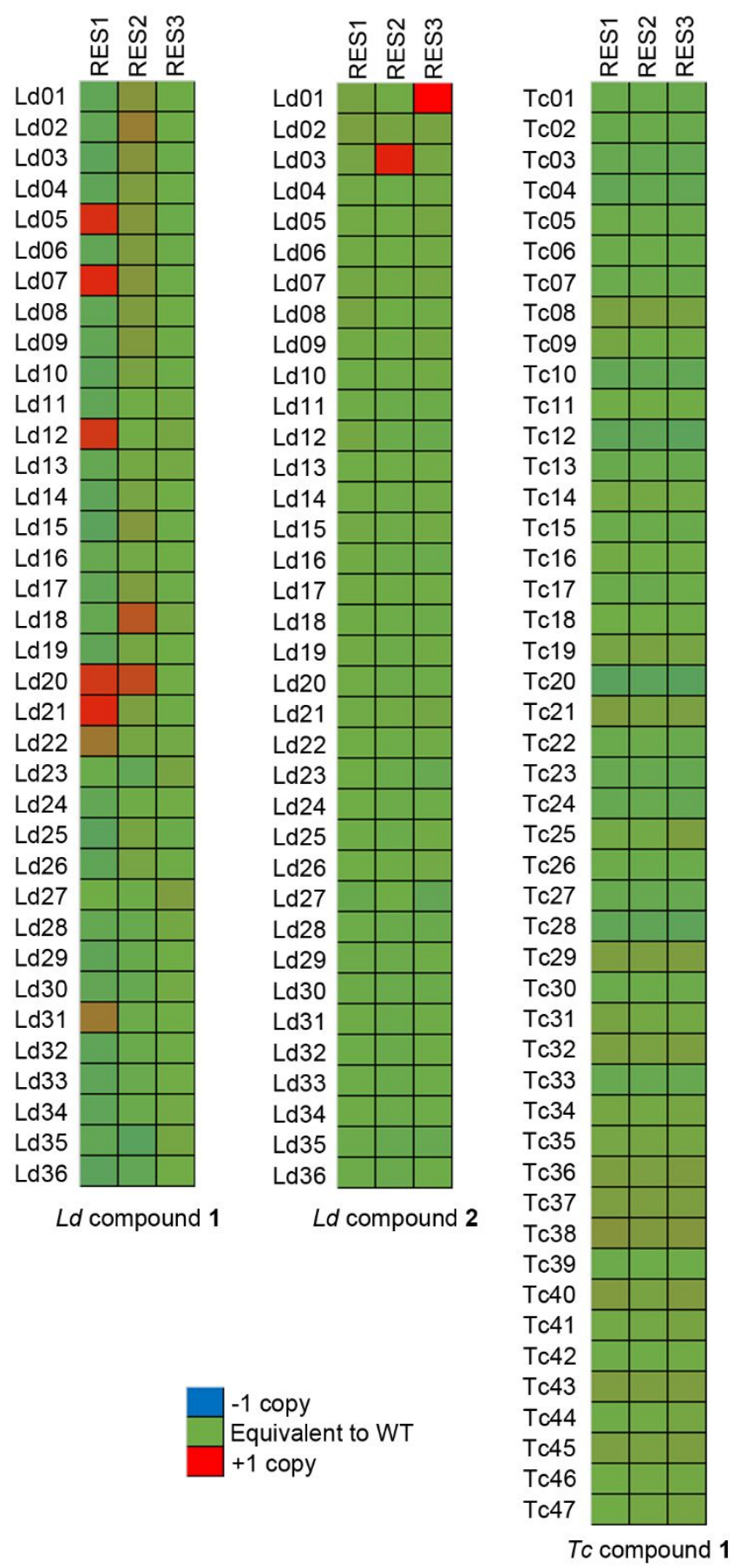

Figure S2 - Copy number variation (CNV) analysis for $L$. donovani $(L d)$ and $T$. cruzi $(T c)$ clones resistant to compounds 1 and compound 2. 

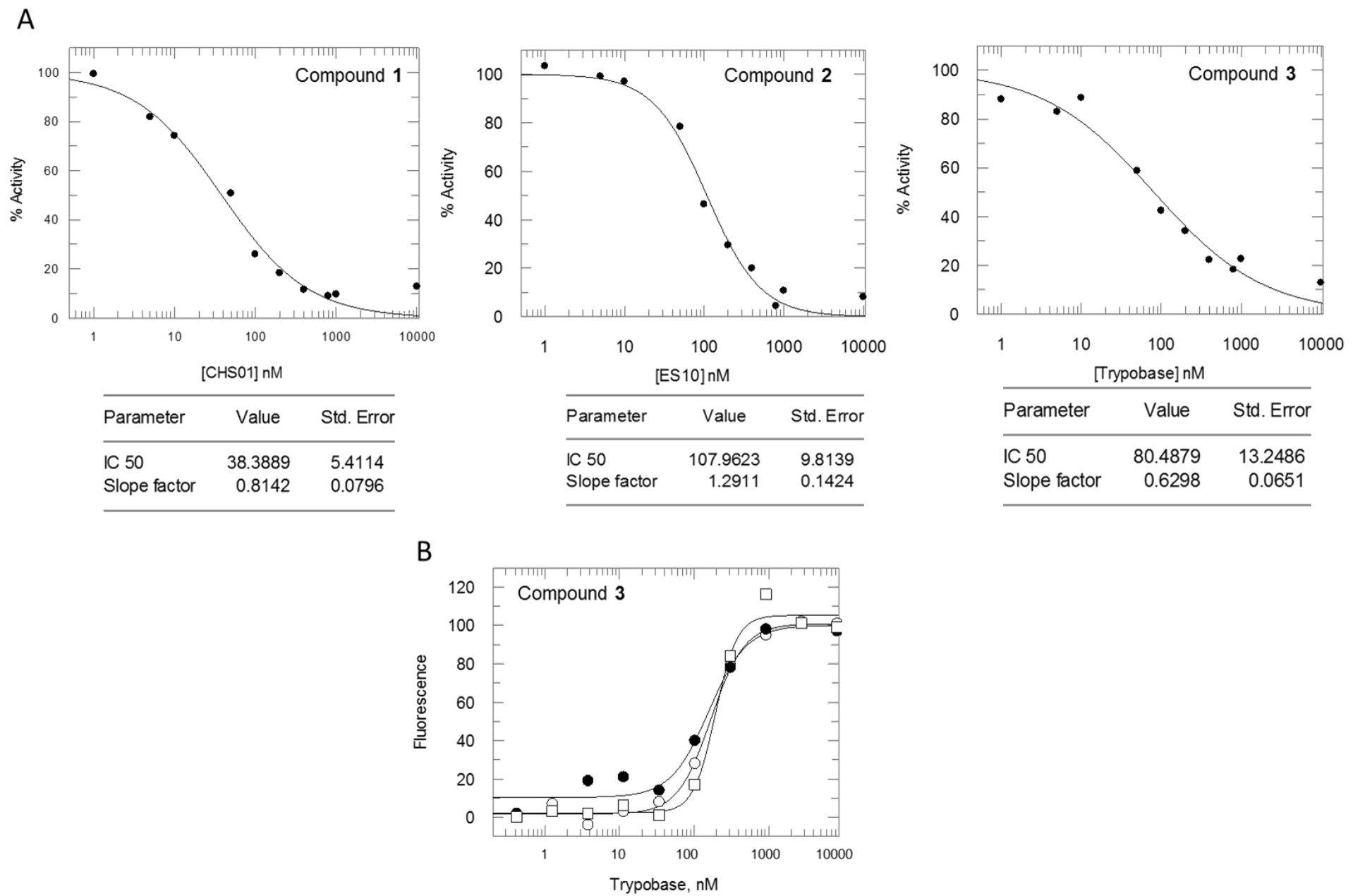

Figure S3 - Representative complex III and $\mathrm{O}_{2}$ consumption assay data. (A) Representative individual IC $\mathrm{C}_{50}$ curves generated from complex III assays (see Materials and Methods for details). The data shown for compounds 1 and 2 were determined in L. donovani lysate. Data shown for compound 3 was determined in $T$. cruzi lysate. (B) Representative $I_{50}$ curves generated in $\mathrm{O}_{2}$ consumption assays with $T$. cruzi epimastigotes (see Materials and Methods for details). Data represents three biological replicates. 


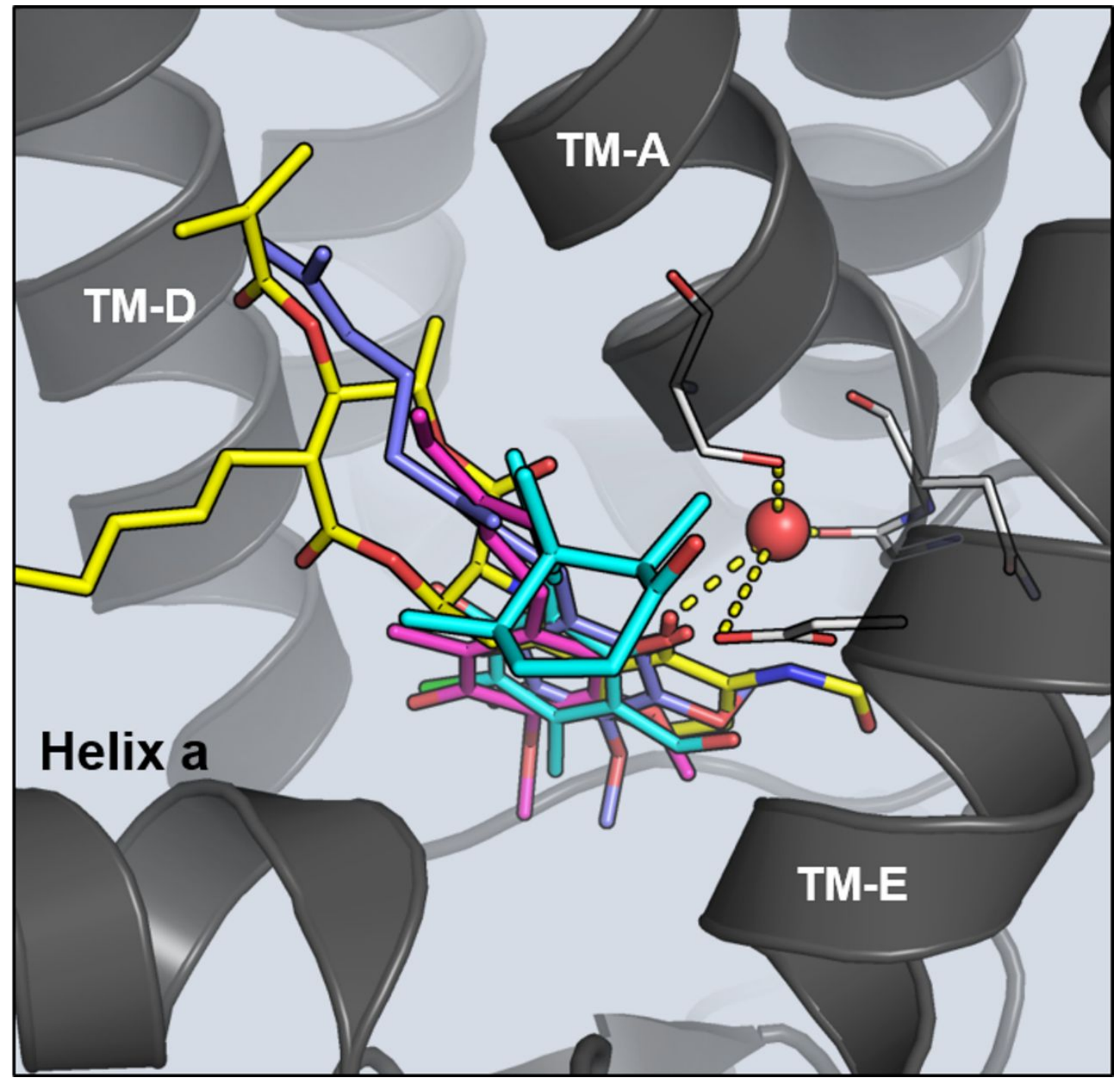

Figure S4 - Conserved water molecule in different X-ray structures of cytochrome $b$ in complex with ligands binding to the $Q_{i}$ site. The water molecule (red sphere) was explicitly considered during the docking calculations of antimycin $A$ and compounds 1, 2 and 3 . The ligands are displayed as sticks: ubiquinone (violet and magenta, PDB 1NTZ and 3L70); ascochlorin (cyan, PDB 3H1L); antimycin A (yellow, PDB ID 1PPJ). 


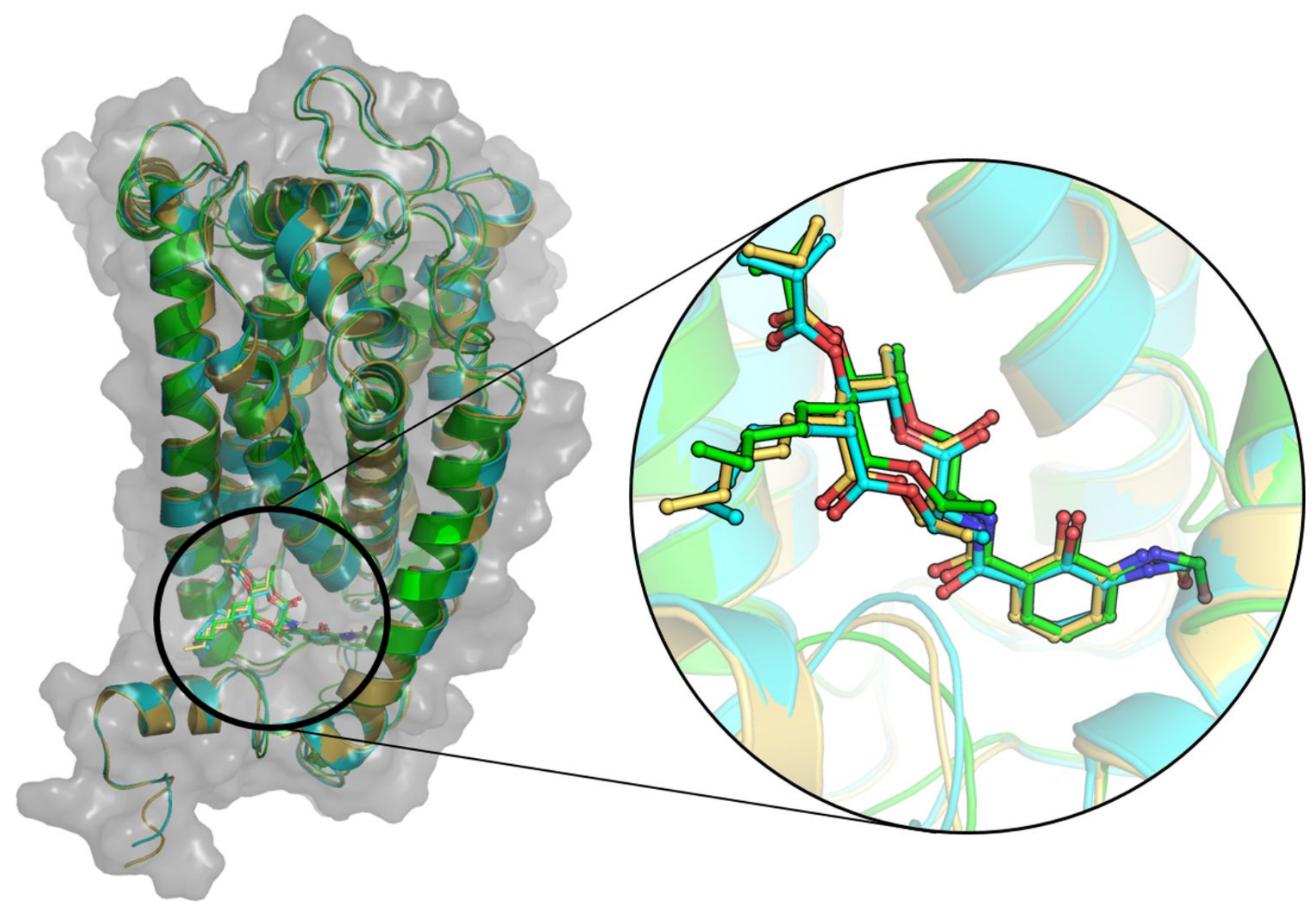

Figure S5 - Binding mode of antimycin A in the cytochrome $b Q_{i}$ site.

Left. Structural alignment of the X-ray crystal structure of cytochrome $b$ from chicken (PDB ID 3H1I, in green) with the homology models of cytochrome $b$ from $L$. donovani (yellow) and $T$. cruzi (cyan). Right. Close-up view into the $Q_{i}$ site of cytochrome $b$ displaying antimycin A (in sticks): avian (in green), L. donovani complex model (in yellow) and T. cruzi complex model (in cyan). 
A

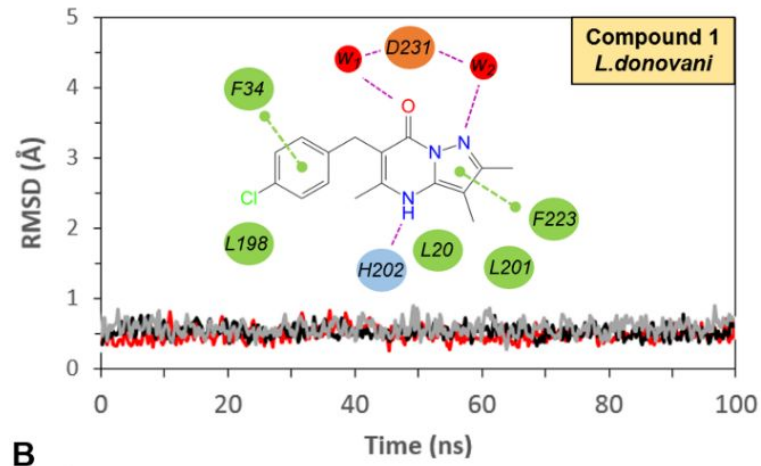

B

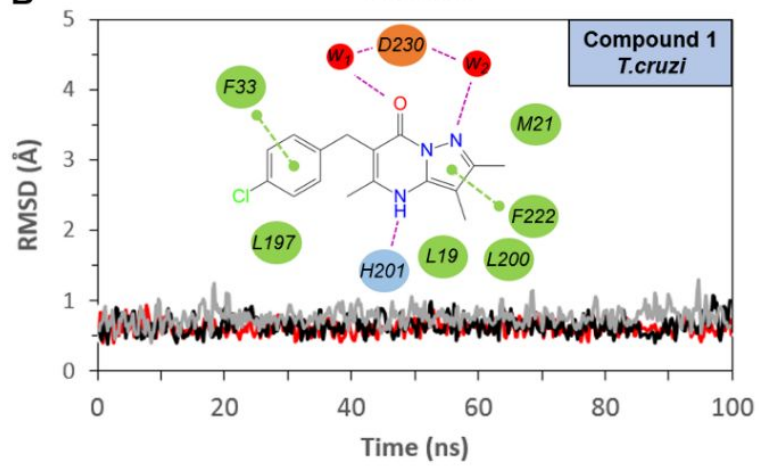

C

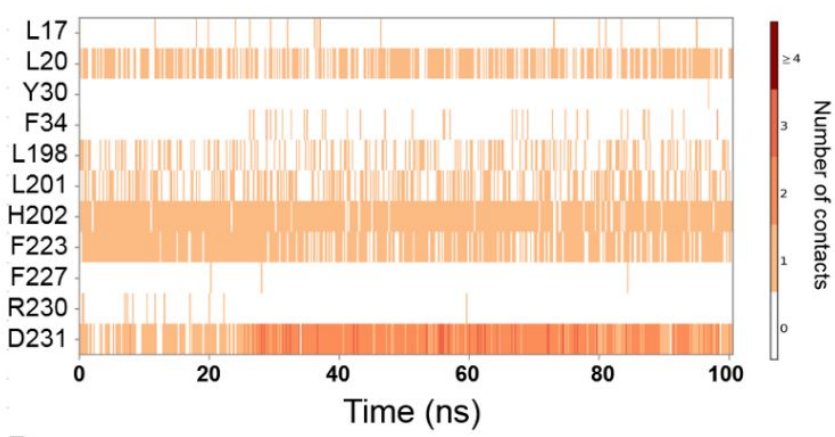

D

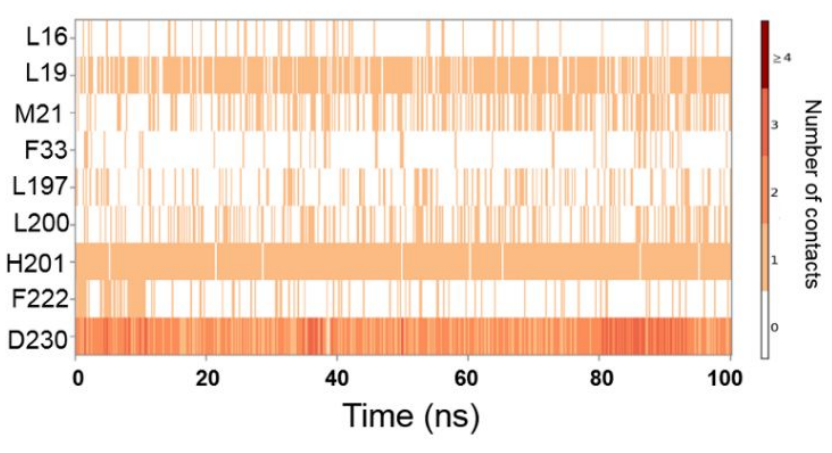

Figure S6 - Structural analysis of compound 1 - cytochrome $\boldsymbol{b}$ interactions. (A and B) Time evolution of the ligand root mean square deviation (RMSD) during the MD simulation of compound 1 bound to the $Q_{i}$ site of cytochrome $b$ from $L$. donovani $(A)$ and $T$. cruzi $(B)$. Each plot corresponds to an independent replica (red, black and grey lines). The most relevant ligand-protein interactions are displayed above the RMSD plot. Hydrophobic residues are highlighted in green, acidic residues in orange, polar residues in blue and water molecules in red. ( $C$ and $D$ ) Time evolution of key ligand-protein contacts during the MD simulations of the cytochrome $b$ from $L$. donovani (C) and T. cruzi (D) in complex with compound 1. 
A

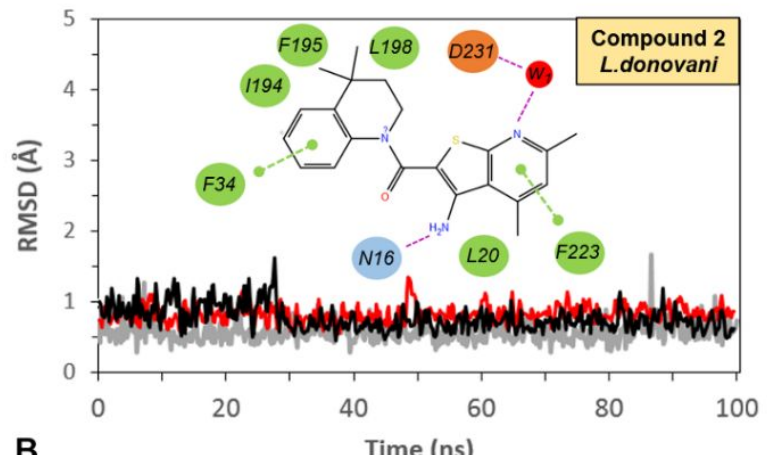

B

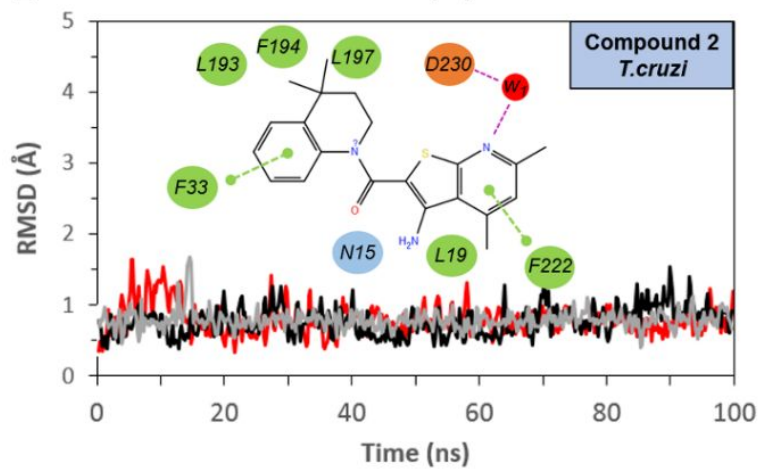

C

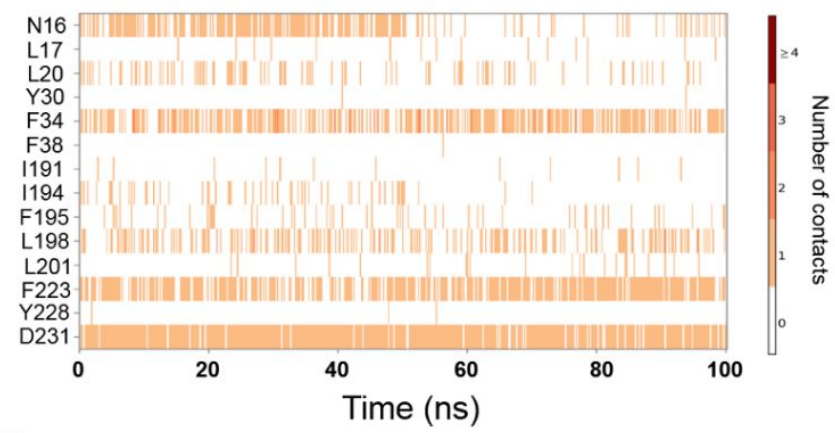

D

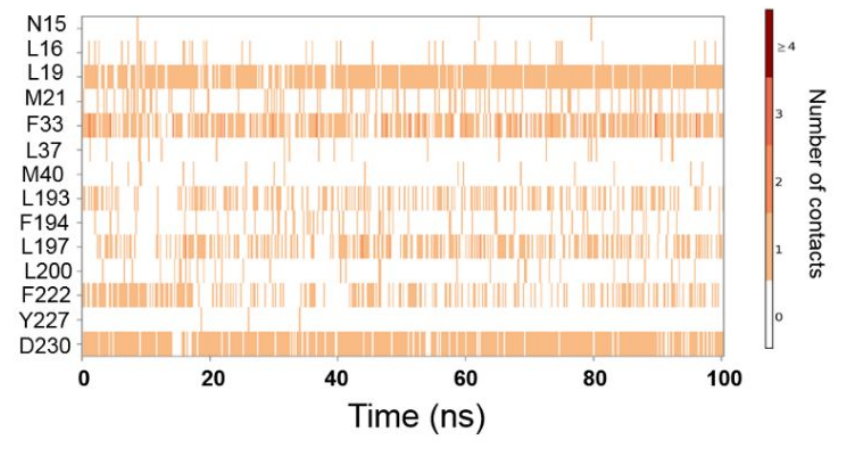

Figure S7 - Structural analysis of compound 2 - cytochrome $\boldsymbol{b}$ interactions. (A and B) Time evolution of the ligand root mean square deviation (RMSD) during the MD simulation of compound 2 bound to the $Q_{i}$ site of cytochrome $b$ from $L$. donovani $(A)$ and $T$. cruzi $(B)$. Each plot corresponds to an independent replica (red, black and grey lines). The most relevant ligand-protein interactions are displayed above the RMSD plot. Hydrophobic residues are highlighted in green, acidic residues in orange, polar residues in blue and water molecules in red. (C and D) Time evolution of key ligand-protein contacts during the MD simulations of the cytochrome $b$ from $L$. donovani (C) and T. cruzi (D) in complex with compound 2. 
A
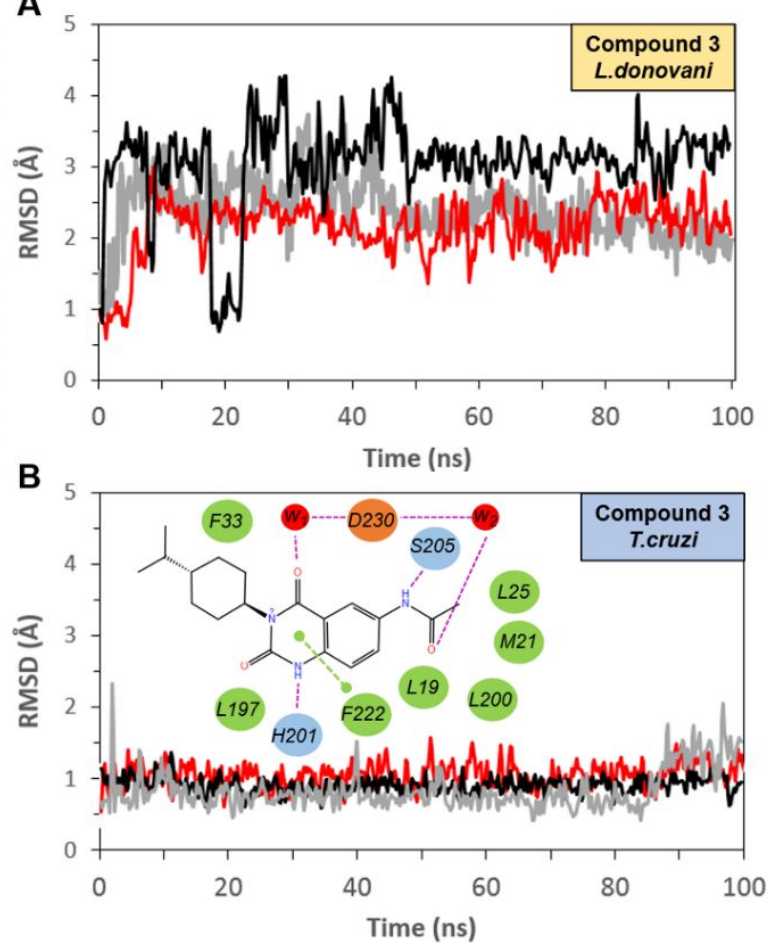

C

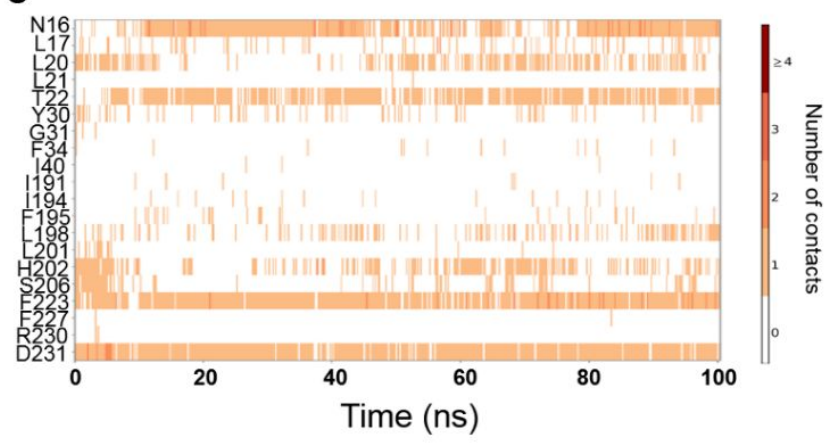

D

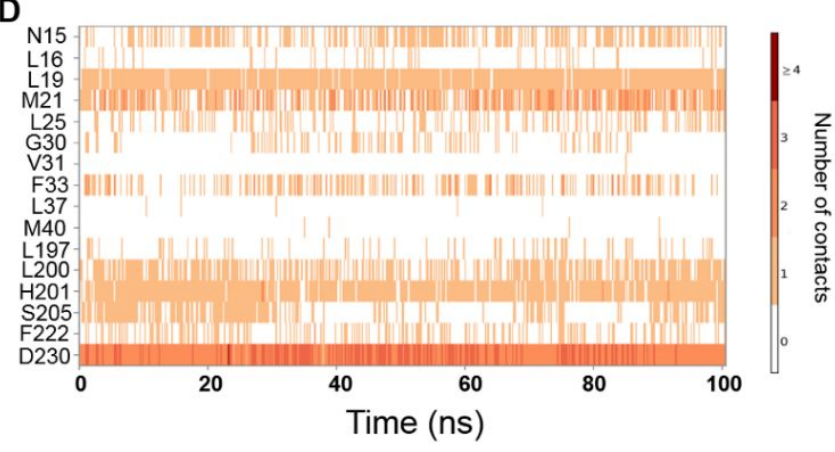

Figure S8 - Structural analysis of compound 3 - cytochrome $\boldsymbol{b}$ interactions. Time evolution of the ligand root mean square deviation (RMSD) during the MD simulation of compound 3 bound to the $Q_{i}$ site of cytochrome $b$ from $L$. donovani $(A)$ and $T$. cruzi $(B)$. Each plot corresponds to an independent replica (red, black and grey lines). The most relevant ligand-protein interactions are displayed above the RMSD plot. Hydrophobic residues are highlighted in green, acidic residues in orange, polar residues in blue and water molecules in red. (C and D) Time evolution of key ligand-protein contacts during the MD simulations of cytochrome $b$ from $L$. donovani $(C)$ and $T$. cruzi (D) in complex with compound 3. 


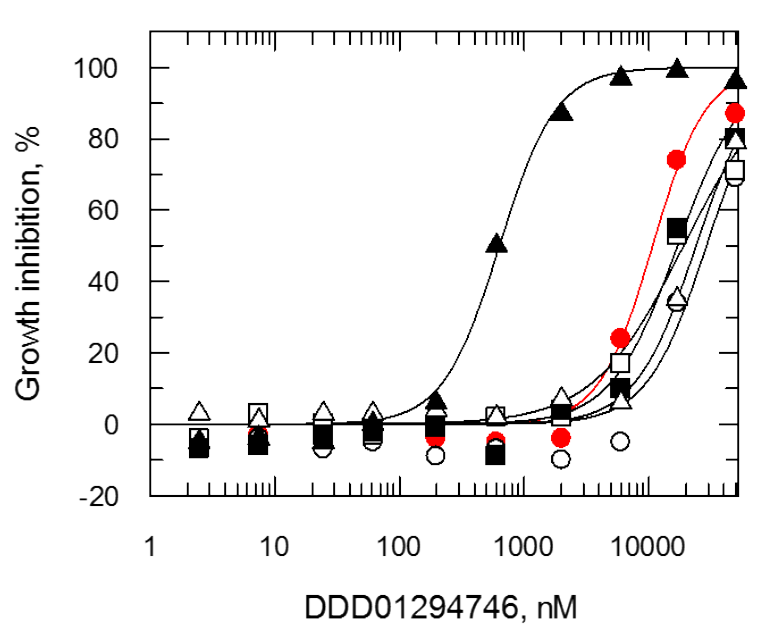

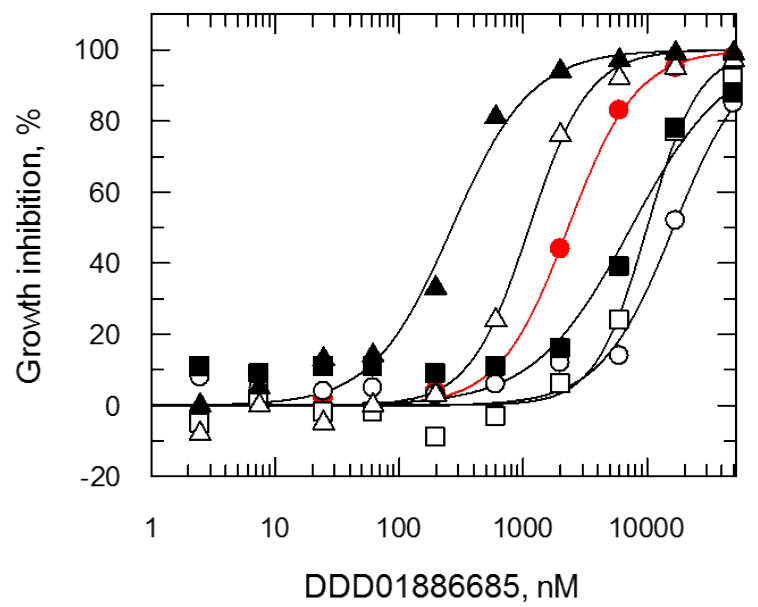

iii

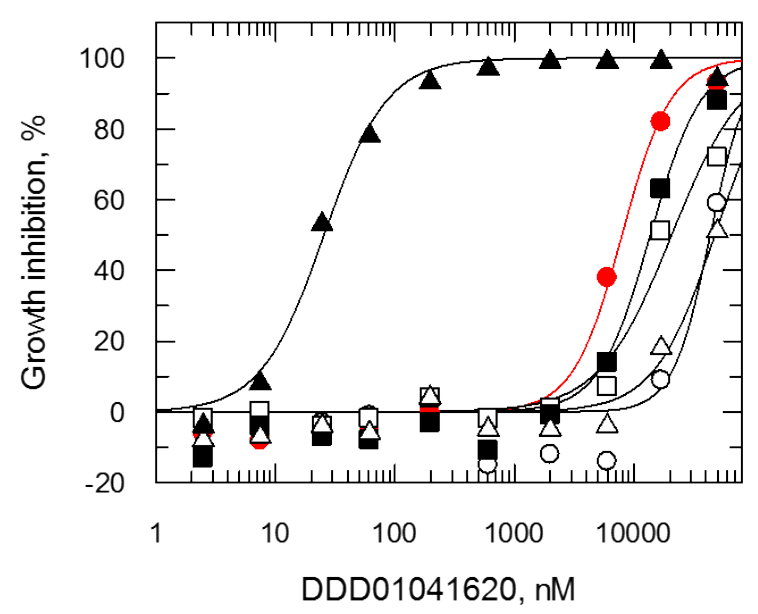

Figure S9 - Cytochrome $\boldsymbol{b}$ resistant panel screen. Test compounds were screened against wild-type T. cruzi epimastigotes (red) and also five cell lines bearing representative mutations within cytochrome $b$. Resistant line R1, open circles; R2, open squares; R3, closed squares; R4, open triangles and R5, closed triangles. Data represents the mean of duplicate technical replicates. 


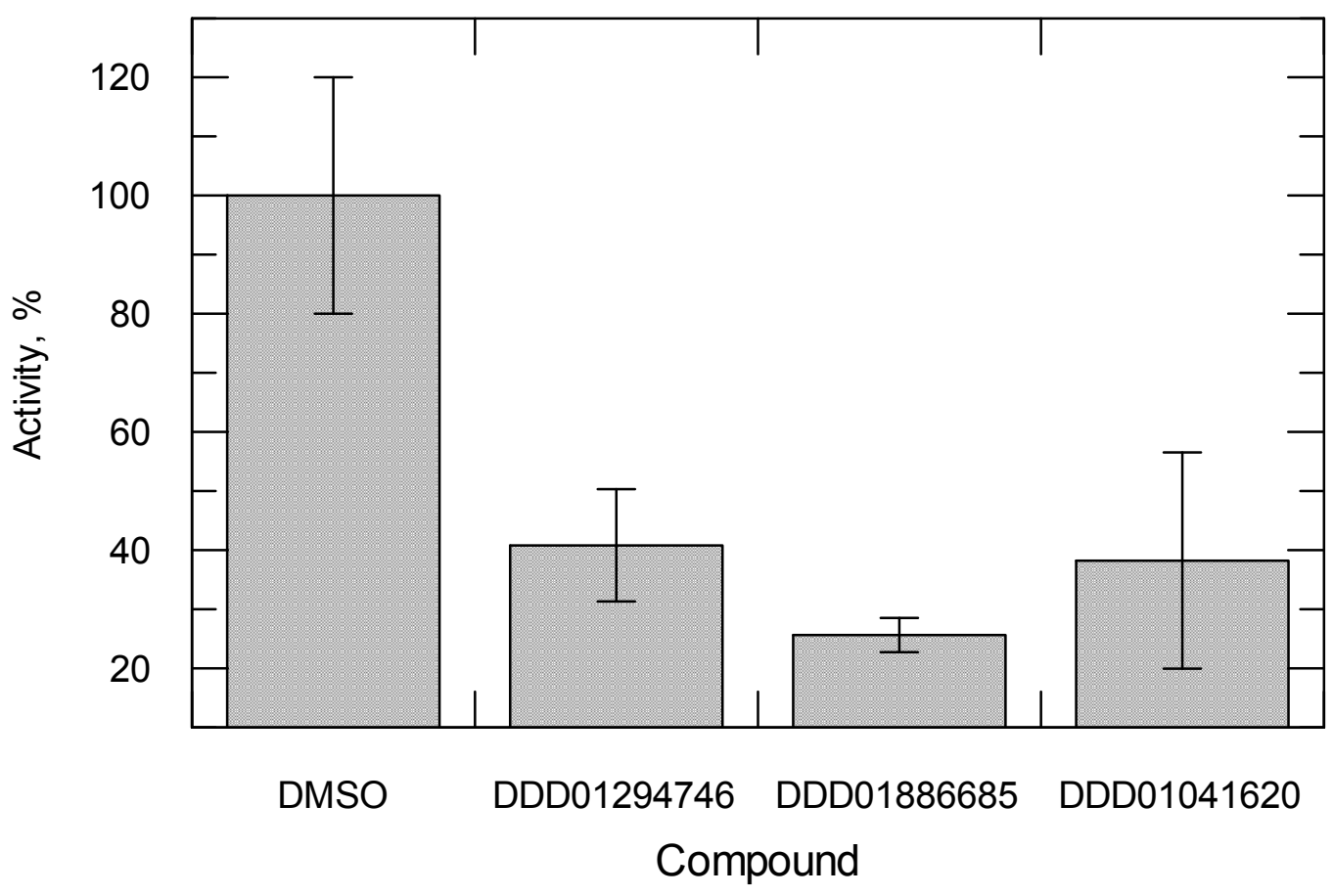

Figure S10 - Complex III assays with compounds identified via the cytochrome $b$ resistant panel screen. Lysates enriched with mitochondria isolated from $T$. cruzi epimastigotes were incubated with test compounds $(20 \mu \mathrm{M})$ or DMSO for 8 min prior to initiation of the complex III assay by the addition of the pseudo substrate decylubiquinol. Complex III activity in the presence of test compounds was determined and compared to activity in the presence of DMSO. Data represents the weighted mean \pm standard deviation of triplicate technical replicates and is representative of the data from three biological replicates. 
Table S1 - Whole genome sequencing for $L$. donovani clones resistant to compound 1 . Summary of read counts and coverage of sequencing (i). Summary of significant SNPs identified in compound 1-resistant clones (ii).

\begin{tabular}{ccccccc}
\hline Cell line & $\begin{array}{c}\text { Number } \\
\text { of reads }\end{array}$ & $\begin{array}{c}\text { Read } \\
\text { length }\end{array}$ & $\begin{array}{c}\text { Percentage } \\
\text { mapped }\end{array}$ & $\begin{array}{c}\text { Fold } \\
\text { coverage }\end{array}$ & $\begin{array}{c}\text { Gain of SNP } \\
\text { heterozygosity }\end{array}$ & $\begin{array}{c}\text { Gain of SNP } \\
\text { homozygosity }\end{array}$ \\
\hline WT & 36675180 & 100 & 87.98 & 88 & - & - \\
RES1 & 36620390 & 100 & 84.93 & 85 & 1 & 1 \\
RES2 & 36764126 & 100 & 86.46 & 87 & 1 & 1 \\
RES3 & 36619010 & 100 & 85.05 & 85 & 1 & 1 \\
\hline
\end{tabular}

ii

\begin{tabular}{|c|c|c|c|c|c|c|c|c|}
\hline \multirow{2}{*}{ Chromosome } & \multirow{2}{*}{$\begin{array}{c}\text { Nucleotide } \\
\text { position }\end{array}$} & \multirow{2}{*}{ Reference } & \multirow{2}{*}{ Mutation } & \multirow{2}{*}{$\begin{array}{c}\text { Amino acid } \\
\text { change }\end{array}$} & \multicolumn{3}{|c|}{ Compound 1} & \multirow{2}{*}{ Gene name } \\
\hline & & & & & $\mathbf{R} 1$ & $\mathbf{R 2}$ & R3 & \\
\hline \multicolumn{9}{|c|}{ Homozygous mutations (coding regions only) } \\
\hline Kinetoplast & 9139 & G & C & Gly37Ala & $1 / 1$ & $1 / 1$ & $0 / 0$ & cytochrome $b$ \\
\hline Kinetoplast & 9694 & G & $\mathrm{T}$ & Cys222Phe & $0 / 0$ & $0 / 0$ & $1 / 1$ & cytochrome $b$ \\
\hline \multicolumn{9}{|c|}{ Heterozygous mutations (coding regions only) } \\
\hline 24 & 688650 & C & $\mathrm{G}$ & Arg565Pro & $0 / 1$ & $0 / 0$ & $0 / 0$ & LdBPK_241820.1; SET domain containing protein \\
\hline 25 & 378147 & $\mathrm{C}$ & A & Gln381Lys & $0 / 0$ & $0 / 0$ & $0 / 1$ & LdBPK_251030.1; hypothetical \\
\hline 35 & 1178869 & C & A & Ala125Ser & $0 / 0$ & $0 / 1$ & $0 / 0$ & LdBPK_352870.1; major facilitator superfamily \\
\hline
\end{tabular}


Table S2 - Whole genome sequencing for T. cruzi clones resistant to compound 1. Summary of read counts and coverage of sequencing (i). Summary of significant SNPs identified in compound 1-resistant clones (ii).

(i)

\begin{tabular}{ccccccc}
\hline Cell line & $\begin{array}{c}\text { Number } \\
\text { of reads }\end{array}$ & $\begin{array}{c}\text { Read } \\
\text { length }\end{array}$ & $\begin{array}{c}\text { Percentage } \\
\text { mapped }\end{array}$ & $\begin{array}{c}\text { Fold } \\
\text { coverage }\end{array}$ & $\begin{array}{c}\text { Gain of SNP } \\
\text { heterozygosity }\end{array}$ & $\begin{array}{c}\text { Gain of SNP } \\
\text { homozygosity }\end{array}$ \\
\hline WT & 36050370 & 100 & 46.09 & 46 & - & - \\
RES1 & 36359988 & 100 & 58.48 & 58 & 1 & 1 \\
RES2 & 36353662 & 100 & 64.19 & 64 & 0 & 1 \\
RES3 & 36438792 & 100 & 59.48 & 59 & 0 & 1 \\
\hline
\end{tabular}

(ii)

\begin{tabular}{|c|c|c|c|c|c|c|c|c|}
\hline \multirow{2}{*}{ Chromosome } & \multirow{2}{*}{$\begin{array}{l}\text { Nucleotide } \\
\text { position }\end{array}$} & \multirow[b]{2}{*}{ Reference } & \multirow[b]{2}{*}{ Mutation } & \multirow{2}{*}{$\begin{array}{l}\text { Amino } \\
\text { acid } \\
\text { change }\end{array}$} & \multicolumn{3}{|c|}{ Compound 1} & \multirow{2}{*}{ Gene name } \\
\hline & & & & & $\mathbf{R} 1$ & $\mathbf{R 2}$ & R3 & \\
\hline \multicolumn{9}{|c|}{ Homozygous mutations (coding regions only) } \\
\hline Kinetoplast & 4742 & C & $\mathrm{T}$ & Leu197Phe & $1 / 1$ & $1 / 1$ & $1 / 1$ & cytochrome $b$ \\
\hline \multicolumn{9}{|c|}{ Heterozygous mutations (coding regions only) } \\
\hline Kinetoplast & 2098 & C & $\mathrm{T}$ & - & $0 / 1$ & $0 / 0$ & $0 / 0$ & NADH dehydrogenase subunit 8 (ND8) pre-edit \\
\hline
\end{tabular}


Table S3 - Assessment of compounds 1 and 2 in complex III assays with lysates prepared from WT and resistant $L$. donovani cell lines. Lysates enriched with mitochondria isolated from $T$. cruzi epimastigotes were incubated with test compounds $(20 \mu \mathrm{M})$ or DMSO for 8 min prior to initiation of the complex III assay by the addition of the pseudo substrate decylubiquinol. Complex III activity in the presence of test compounds was determined and compared to activity in the presence of DMSO. Fold-changes in sensitivity compared to wildtype are in parentheses.

\begin{tabular}{|c|c|c|}
\hline \multirow{2}{*}{ Cell lines } & \multicolumn{2}{|c|}{$\mathrm{IC}_{50}$ values, $\mathrm{nM}$} \\
\cline { 2 - 3 } & Compound 1 & Compound 2 \\
\hline WT & $44 \pm 7$ & $99 \pm 13$ \\
\hline Compound 1 RES1 & $220 \pm 51(5)$ & $4200 \pm 500(42)$ \\
\hline Compound 2 RES3 & & \\
\hline
\end{tabular}

Data represents the weighted mean \pm SD of at least three biological replicates $(n \geq 3)$. 
Table S4 - Whole genome sequencing for $L$. donovani clones resistant to compound 2 . Summary of read counts and coverage of sequencing (i). Summary of significant SNPs identified in compound 2-resistant clones (ii).

(i)

\begin{tabular}{ccccccc}
\hline Cell line & $\begin{array}{c}\text { Number } \\
\text { of reads }\end{array}$ & $\begin{array}{c}\text { Read } \\
\text { length }\end{array}$ & $\begin{array}{c}\text { Percentage } \\
\text { mapped }\end{array}$ & $\begin{array}{c}\text { Fold } \\
\text { coverage }\end{array}$ & $\begin{array}{c}\text { Gain of SNP } \\
\text { heterozygosity }\end{array}$ & $\begin{array}{c}\text { Gain of SNP } \\
\text { homozygosity }\end{array}$ \\
\hline WT & 38367592 & 100 & 81.37 & 86 & - & - \\
RES1 & 35899862 & 100 & 82.41 & 81 & 1 & 1 \\
RES2 & 36102782 & 100 & 80.19 & 79 & 0 & 1 \\
RES3 & 35915250 & 100 & 83.71 & 82 & 0 & 1 \\
\hline
\end{tabular}

(ii)

\begin{tabular}{|c|c|c|c|c|c|c|c|c|}
\hline \multirow{2}{*}{ Chromosome } & \multirow{2}{*}{$\begin{array}{l}\text { Nucleotide } \\
\text { position }\end{array}$} & \multirow{2}{*}{ Reference } & \multirow{2}{*}{ Mutation } & \multirow{2}{*}{$\begin{array}{l}\text { Amino acid } \\
\text { change }\end{array}$} & \multicolumn{3}{|c|}{ Compound 2} & \multirow{2}{*}{ Gene name } \\
\hline & & & & & R1 & $\mathbf{R 2}$ & R3 & \\
\hline \multicolumn{9}{|c|}{ Homozygous mutations (coding regions only) } \\
\hline Kinetoplast & 9121 & G & C & Gly31Ala & $0 / 0$ & $1 / 1$ & $0 / 0$ & cytochrome $b$ \\
\hline Kinetoplast & 9648 & $\mathrm{~T}$ & C & Ser207Pro & $1 / 1$ & $0 / 0$ & $0 / 0$ & cytochrome $b$ \\
\hline Kinetoplast & 9708 & $\mathrm{~T}$ & A & Phe227lle & $0 / 0$ & $0 / 0$ & $1 / 1$ & cytochrome $b$ \\
\hline \multicolumn{9}{|c|}{ Heterozygous mutations (coding regions only) } \\
\hline 4 & 1272440 & G & A & Cys179Tyr & $0 / 1$ & $0 / 0$ & $0 / 0$ & LdBPK_150110.1; hypothetical \\
\hline
\end{tabular}


Table S5 - Assessment of compound potency against wild-type and resistant $L$. donovani cell lines in intra-macrophage assays. The potency of compounds 1 and $\mathbf{2}$ were assessed against WT parasites and also resistant clones generated by exposure to compounds 1 and 2. Compound DDD01012232, an established divalent cation chelator [58], was also assessed as a negative control. Cell lines demonstrating resistance or crossresistance relative to wild-type are highlighted in blue while those demonstrating hypersensitivity are highlighted in grey. Fold-changes in sensitivity compared to wild-type are in parentheses.

\begin{tabular}{|c|c|c|c|}
\hline \multirow{2}{*}{ Compound ID } & \multicolumn{3}{|c|}{ Leishmania EC $_{50}, \mu \mathrm{M}$} \\
\cline { 2 - 4 } & WT & Compound 1 RES1 & Compound 2 RES1 \\
\hline DDD01012232 (CON) & 0.1 & 0.1 & 0.2 \\
\hline Compound 1 & 0.1 & $1.3(11)$ & $0.02(6)$ \\
\hline Compound 2 & 0.4 & $0.03(13)$ & $4.4(11)$ \\
\hline
\end{tabular}

Data represents the mean \pm SD of at least two technical replicates. 